\title{
Comparison of Antibiotic Dosing Before and After Implementation of an Electronic Order Set
}

\author{
Kristen R. Nichols ${ }^{1}$ Allison L. Petschke ${ }^{1} \quad$ Emily C. Webber $^{2} \quad$ Chad A. Knoderer $^{1}$ \\ 1 Pharmacy Practice Department, Butler University College of \\ Pharmacy and Health Sciences, Indianapolis, Indiana, United States \\ 2 Department of Pediatrics, Indiana University School of Medicine, \\ Indianapolis, Indiana, United States \\ Address for correspondence Kristen R. Nichols, PharmD, Pharmacy \\ Practice Department, Butler University College of Pharmacy and \\ Health Sciences, 4600 Sunset Avenue, Indianapolis, IN 46208, \\ United States (e-mail: knichols@butler.edu).
}

Appl Clin Inform 2019;10:229-236.

\begin{abstract}
Keywords

- antibiotic stewardship

- clinical decision support

- inpatient CPOE

- order set

- pediatrics

Background To maximize resources, the antimicrobial stewardship program at a pediatric tertiary care hospital made pediatric dosing specific guidance within the electronic health record available to all hospitals within the health system.

Objective The objective of this study was to compare the appropriateness of antibiotic dosing before and after the implementation of an electronic intravenous (IV) antibiotic order set.

Methods This was a retrospective cohort study evaluating orders from patients younger than 18 years who received cefepime, piperacillin-tazobactam, tobramycin, or gentamicin at 12 health-system hospitals. Antibiotic dosing regimens and order set use were evaluated in patients who received the specified antibiotics during the 6month time frame prior to and following electronic order set availability at each hospital.

Results In the before and after implementation periods, 360 and 387 total antibiotic orders were included, respectively. Most orders were gentamicin (55.8\% in the before implementation period and $54.5 \%$ in the after implementation period) followed by piperacillin-tazobactam ( $22.5 \%$ in the before period and $22.2 \%$ in the after period). Overall, 663 orders were classified as appropriate (88.8\%). Appropriateness was similar in the before or after implementation periods ( $87.8 \mathrm{vs.} 89.7 \%, p=0.415)$. There was a significant difference in appropriateness if a blank order versus the electronic IV antibiotic order set was used (82.8 vs. $90.5 \% ; p=0.024)$.

Conclusion No difference in antibiotic appropriateness overall was found in the before and after implementation periods. However, when specifically compared with the appropriateness of dosing when blank order forms were used, dosing was more appropriate when electronic antibiotic order sets were used.
\end{abstract}

\section{Background and Significance}

Physical growth, development, and the requirement for weight-based dosing increase vulnerability of pediatric patients to medication errors, which can lead to serious adverse consequences. ${ }^{1}$ Tenfold dosing errors are most likely to occur because of an error in the prescribing step of the medication use process, as compared with transcription or administration, and antibiotics have been associated with both over- and underdosing errors. ${ }^{2}$ Antibiotics were found to be the most common drug class implicated in medication-related visits to emergency departments, and received

November 16, 2018

accepted after revision

February 8, 2019
(C) 2019 Georg Thieme Verlag KG Stuttgart . New York
DOI https://doi.org/

10.1055/s-0039-1683877. ISSN 1869-0327. 
incorrect dosing was the reason for $30 \%$ of medication errors overall. ${ }^{3}$

The implementation of computerized physician order entry (CPOE) and various associated clinical decision support functionalities have, in general, been found to reduce the incidence of many types of medication errors in pediatrics, though findings specifically with respect to dosing improvement have been mixed. ${ }^{4}$ Electronic order sets have been associated with desired changes in treatment choice and monitoring, including antibiotic choices and decreasing time to receipt of antibiotics. ${ }^{5-12}$ Prewritten electronic order sentences, such as those included in electronic order sets, may be less cumbersome than the use of dosing alerts for optimizing medication dosing. ${ }^{13-16}$ When well designed, CPOE functions have been shown to support institutional antimicrobial stewardship activities. ${ }^{11-13,16-18}$ However, CPOE may be associated with negative consequences including medication errors and increased time burden. A change to electronic from paper ordering should not be assumed successful without evaluation. ${ }^{19,20}$ The extension of resources to distant sites through electronic CPOE means is limited in pediatrics. ${ }^{7,8}$ One study found a decrease in narcotic prescribing across many Kaiser Permanente locations with electronic health record (EHR) restrictions, and another demonstrated an improvement in chosen imaging modalities for pediatric appendicitis at community hospitals secondary to an electronic order set shared from a tertiary pediatric academic hospital. ${ }^{7,8}$ Neither of these focused on the facilitation of appropriate dosing regimens in pediatric antimicrobial stewardship (PAS).

Our health system consists of 1 pediatric tertiary care hospital and 11 additional affiliated system hospitals that may provide care for pediatric patients. PAS services exist solely at the pediatric hospital. Optimized antibiotic dosing initiatives are made available to other system hospitals in an effort to share expertise in antibiotic dosing. In 2008, the antibiotic stewardship program at our pediatric tertiary care hospital created an extensive paper order set that was required for intravenous (IV) antibiotic orders. This form provided dosing recommendations based on published data and pharmacokinetic and pharmacodynamic principles. Prior to the development of the PAS and paper order set, prescribers at the pediatric and system hospitals ordered antibiotics using a plain/blank order sheet (essentially a lined piece of paper). The paper order set was mandatory for use at the pediatric hospital beginning in 2010, but at system hospitals, prescribers could choose to continue to use either a blank order sheet or the paper order set for antibiotics. At the pediatric hospital, the paper order set improved the accuracy for the initial antibiotic dose, interval, and overall order compared with the blank order sheet. ${ }^{21}$

In 2012, during the health system's transition to CPOE, electronic order sets for IV antibiotics were created for mandatory use at the pediatric hospital. ${ }^{22}$ Additional decision support detail was provided in the electronic order sets compared with the previous paper order sets. Electronic order sets were also embedded in diagnosis-specific order sets and affiliated with communication tools to promote stewardship and approval processes. All IV antibiotic electronic order sets were made available to other system hospitals, but they were only required at the main pediatric hospital. The usage and impact of these electronic order sets on appropriate antibiotic dosing are unknown. At the time of the order set implementation, it was hypothesized that dosing, specifically at other system hospitals, would be improved.

\section{Objective}

The objective of this study was to compare the appropriateness of antibiotic dosing before and after the availability of IV antibiotic electronic order sets.

\section{Methods}

A retrospective cohort study using data from electronic medical records (EMRs) was performed. Orders for IV antibiotics commonly used in our health system, which included IV cefepime, gentamicin, piperacillin-tazobactam, and tobramycin, were chosen for evaluation. These antibiotics were chosen by the research team based on order volume and the potential for suboptimal dosing. A convenience sample of eligible orders was identified using a pharmacy computer system generated report of patients younger than 18 years with orders for the selected antibiotics within the time frame of 6 months prior and 6 months following electronic order set availability. Verbal and telephone orders were excluded based on local practice to minimize the impact that a pharmacist could have had on those orders and to focus more on prescriber-ordered doses. Orders for antibiotics intended for prophylaxis, defined as the receipt of antibiotic therapy for the prevention of surgical site infection during the perioperative period, which included 24 hours preceding and following surgical intervention, were also excluded. Orders for one-time antibiotic doses without specified frequencies were excluded unless an additional subsequent order for the same antibiotic contained a dose and frequency.

The number of pediatric patients cared for by the system hospitals and the number of orders at each system hospital varied. To allow for a comprehensive analysis of each order, a prespecified number of antibiotic orders comprising the sample that was analyzed from each hospital was determined based on the volume of orders identified preliminarily ( $\sim$ Table 1 ). Identified orders were separated by month and randomized using Excel, after which data were collected for the number of prespecified orders at each hospital.

The primary outcome was antibiotic dosing appropriateness. Secondary outcomes included the reasons why specific regimens were ruled as inappropriate, for example, wrong frequency or dose higher than adult dose, and comparison of appropriateness between various order types. Order types could include blank paper order sheets (with ruled lines), electronic orders, paper IV antibiotic order set, electronic IV antibiotic order set, or other paper 
Table 1 Strategy for narrowing identified antibiotic orders after randomization

\begin{tabular}{|l|l|}
\hline $\begin{array}{l}\text { Identified orders } \\
\text { per hospital }\end{array}$ & $\begin{array}{l}\text { Orders included } \\
\text { for analysis }\end{array}$ \\
\hline$<50$ & All \\
\hline $51-100$ & $50 \%$ \\
\hline $101-200$ & $40 \%$ \\
\hline $201-300$ & $30 \%$ \\
\hline $301-500$ & $20 \%$ \\
\hline $501-1,000$ & $10 \%$ \\
\hline $1,001-2,000$ & $5 \%$ \\
\hline$>2,001$ & $2.5 \%$ \\
\hline
\end{tabular}

order sets. Outcomes were compared between orders in the periods before and after the availability of the electronic antibiotic order sets. De-identified collection instruments were used in the extraction of data from the EMR. For each order, the dosing regimen, order type, and patient's demographic information were collected. Antibiotic dose and frequency for each patient were assessed for appropriateness, which was determined using prespecified definitions for each antibiotic. These definitions were based on recommended weight-based drug dosing, usual adult doses, primary literature, and pharmacokinetic and pharmacodynamic properties of each drug, and were agreed upon by three of the investigators (A. P., K. N., and C. K.). For example, piperacillin/tazobactam dosed every 8 hours would be considered inappropriate, unless it was administered as an extended infusion, due to the short half-life and time-dependent activity of the drug. A dose exceeding the typical adult dose would also be considered inappropriate. Antibiotic therapy was categorized appropriate based on drug-specific criteria if both the dose and frequency were consistent with the preset criteria. A patient's renal function (estimated glomerular filtration rate) was evaluated using the modified Schwartz equation if a dose or frequency was determined to be inappropriate, and if the dose adjustment was because of diminished renal function, it was classified as appropriate. The online database LexiComp and the text Drug Prescribing in Renal Failure were used for the evaluation of renally adjusted doses. ${ }^{23,24}$ One investigator compared all of the doses extracted from the medical records to the preset criteria. Only the first dosing regimen of an antibiotic was included. If appropriateness was uncertain, two additional investigators would evaluate the case, and the three investigators would reach an agreement.

Statistical analysis was conducted using the Statistical Package for Social Sciences (Version 22, IBM) software. Descriptive statistics were used to characterize the orders and patients. Categorical data, including antibiotic dosing appropriateness, for pre- and postelectronic order set groups were compared using chi-square or Fisher's exact tests as appropriate.

\section{Results}

Initial pharmacy reports contained 6,372 antibiotic orders from all system hospitals within the study timeframe. Those orders were narrowed as described previously, and then each order that met the inclusion criteria was evaluated, resulting in 919 antibiotic orders. Following exclusions, 747 evaluable antibiotic orders were included in the analysis. The most common reason for exclusion was antibiotic administration as a one-time dose without a specific frequency (-Table 2).

- Table 3 displays order characteristics in the before and after periods. More than half of the orders were for patients younger than 3 months both before and after electronic order set implementation (58.1 and 57.6\%). The neonatal intensive care unit was the primary service for about half of the included orders (-Table 3). Age and sex of patients for whom orders were placed were similar in the before and after periods, with $54.4 \%$ of all orders placed for male patients. The highest number of included orders was at the pediatric tertiary care hospital. Specific numbers of antibiotic orders contributed from each hospital are found in - Table 4 .

There was variation in how antibiotics were ordered at different hospitals prior to providing electronic order sets. At the pediatric tertiary hospital, 99\% (107/108) of orders were written on a paper IV antibiotic order set in the before period and $97.4 \%(113 / 116)$ of orders were placed using the electronic order set in the after period. At the other system hospitals, taken as aggregate, only $3.2 \%$ (8/252) of orders were written on the paper IV antibiotic order set supplied by PAS in the before period. The remainder of orders were placed on blank order sheets or various paper order sets. In the after period, 48\% (130/271) were placed using the electronic IV antibiotic order set.

There was no difference in antibiotic dosing appropriateness in the before and after study periods when all orders were combined ( 87.8 vs. $89.7 \% ; p=0.415$ ). Similarly, there was no difference in antibiotic dosing appropriateness when the electronic order set was used versus a combination of any other order format during both study periods ( 90.5 vs. $87.9 \%$; $p=0.285$ ). There was a significant improvement in antibiotic dosing appropriateness when the electronic order set was used versus a blank order sheet at all hospitals during both study periods ( 90.5 vs. $82.8 \% ; p=0.024$ ).

At the pediatric tertiary care hospital specifically, antibiotic orders were as appropriate in the before period as they were in the after implementation period (89.8 vs. $94.8 \%$; $p=0.157$ ). This was consistent when also looking at all other

Table 2 Exclusion reasons for 172 excluded orders

\begin{tabular}{|l|l|}
\hline Reason for exclusion & Frequency, $\boldsymbol{n}(\%)$ \\
\hline One-time dose & $66(38.4)$ \\
\hline Perioperative prophylaxis & $55(32)$ \\
\hline Verbal or telephone order & $48(27.9)$ \\
\hline Per protocol pharmacy dosing & $3(1.7)$ \\
\hline
\end{tabular}


Table 3 Primary ordering service and order types

\begin{tabular}{|c|c|c|}
\hline \multirow[t]{2}{*}{ Primary service } & \multicolumn{2}{|c|}{ Number of orders (\%) } \\
\hline & Before $(n=360)$ & After $(n=387)^{a}$ \\
\hline $\mathrm{NICU}$ & $188(52.2)$ & $198(51.2)$ \\
\hline General medical service & $99(27.5)$ & $130(33.6)$ \\
\hline General surgical service & $36(10)$ & $29(7.5)$ \\
\hline PICU & $31(8.6)$ & $28(7.2)$ \\
\hline ED & $6(1.7)$ & $2(0.5)$ \\
\hline \multicolumn{3}{|l|}{ Order type } \\
\hline Blank order & $98(27.2)$ & $47(12.1)$ \\
\hline Written order set & $190(52.8)$ & $50(12.9)$ \\
\hline CPOE & $27(7.5)$ & $20(5.2)$ \\
\hline Other electronic order set & $45(12.5)$ & $27(7)$ \\
\hline Neonatal IV antibiotic electronic order set & - & $121(31.3)$ \\
\hline Pediatric IV antibiotic electronic order set & - & $122(31.5)$ \\
\hline
\end{tabular}

Abbreviations: CPOE, computerized physician order entry; ED, emergency department; NICU, neonatal intensive care unit; PICU, pediatric intensive care unit.

${ }^{a}$ No statistically significant differences were noted between groups.

system hospitals combined, minus the pediatric hospital (86.9 vs. $87.5 \% ; p=0.851$ ).

Cefepime orders were significantly more appropriate when ordered through the electronic order set versus a blank order sheet ( 92.1 vs. $69.2 \% ; p=0.017$ ) but not when electronic order set orders were compared with any other order format (92.1 vs. $79.7 \% ; p=0.095$ ). Tobramycin orders were also significantly more appropriate when ordered through the electronic order set only when compared with the blank order sheet ( 97.3 vs. $50 \% ; p=0.001$ ). There was no difference in antibiotic dosing appropriateness when the electronic order set was used versus any other order format for gentamicin ( 88.7 vs. $91.9 \% ; p=0.305$ ) or piperacillin-tazobactam ( 88.7 vs. $81.6 \% ; p=0.246$ ). This finding held true when specifically comparing electronic order set to blank order sheet (gentamicin 88.7 vs. $89.9 \%, p=0.795$; piperacillin-tazobactam $88.7 \%$ vs. $80.6 \%, p=0.287$ ).
Eighty-four antibiotic orders were classified as inappropriate overall. On blank order forms, the most common reason for classification as inappropriate was traditional dosing of aminoglycosides in $4 / 84$ orders (4.8\%). The most common reason for classification as inappropriate when reviewing written order sets was a dose that exceeded appropriate maximum based on either adult or indication specific dosing in 6/84 orders (7.1\%). On electronic orders in CPOE (without any dosing guidance), the most common reason for classification as inappropriate was the piperacillin-tazobactam interval administered every 8 hours, but not infused over 4 hours, in 5/84 orders (6\%). On the neonatal electronic order sets, the most common reason for classification as inappropriate was too frequent dosing interval in 12 orders (14.2\%). Gentamicin or tobramycin accounted for all 15 of these orders. There was no single most common reason for classification as inappropriate on the pediatric electronic

Table 4 Antibiotic orders contributed to the study from each institution

\begin{tabular}{|c|c|c|c|c|c|}
\hline $\begin{array}{l}\text { De-identified hospital } \\
\text { number }\end{array}$ & Included orders, $n$ (\%) & Gentamicin & Tobramycin & Piperacillin/ tazobactam & Cefepime \\
\hline 1 (pediatric hospital) & $224(30)$ & $56(25)$ & $60(26.8)$ & $66(29.5)$ & $42(18.8)$ \\
\hline 6 & $138(18.5)$ & $69(50)$ & $2(1.4)$ & $38(27.5)$ & $29(21)$ \\
\hline 4 & $119(15.9)$ & $79(66.4)$ & $3(2.5)$ & $19(16)$ & $18(15.1)$ \\
\hline 2 & $101(13.5)$ & $72(71.3)$ & $1(1)$ & $22(21.8)$ & $6(5.9)$ \\
\hline 10 & $59(7.9)$ & $50(84.7)$ & - & $3(5.1)$ & $6(10.2)$ \\
\hline 3 & $56(7.5)$ & $49(87.5)$ & - & $7(12.5)$ & - \\
\hline 11 & $43(5.8)$ & $34(79.1)$ & - & $8(18.6)$ & $1(2.3)$ \\
\hline 12 & $4(0.5)$ & $2(50)$ & - & $2(50)$ & - \\
\hline 7 & $2(0.3)$ & - & - & $2(100)$ & - \\
\hline 9 & $1(0.1)$ & $1(100)$ & - & - & - \\
\hline
\end{tabular}


order sets or any other electronic order set. Reasons for classification as inappropriate varied widely at each system hospital.

\section{Discussion}

With the abundance of antibiotic dosing recommendations and resources available to prescribers, choosing the best dose can be a challenge. Prior to our study, the use of paper order sets with dosing guidance had been shown to improve the initial dose, frequency, and overall accuracy for antibiotic orders as compared with blank order sheets. ${ }^{21}$ It was reasonable to hypothesize that that electronic order sets would improve the initial dose, frequency, and appropriateness of dosing as compared with blank order sheets or electronic ordering with no dosing guidance. However, we observed no overall difference in the appropriateness of antibiotic dose and frequency in the study period before and after the availability of an electronic order set. We specifically evaluated the appropriateness of dosing before and after the availability of electronic order sets in CPOE and not use of CPOE overall, which could explain why these findings contrast with the reduction in total medication errors observed with CPOE. 4,25 Previous studies have demonstrated an increase in appropriate or optimal antibiotic dosing when comparing pre- and postdosing guidance implementation strategies in adults. ${ }^{15,16}$ The fact that the appropriateness of dosing did not decrease after the availability of the order sets is positive and supports continued electronic dosing guidance. A recently published study in adult patients that aimed to use electronic order sets to improve antibiotic choices, dosing, and compliance with hospital antibiotic policies also found no difference in appropriateness. ${ }^{13}$ However, this study was different from ours in that it replaced alerts with order sets, whereas we compared different ordering modalities and continued to have stewardship alerts at the primary pediatric hospital.

It is unsurprising that the electronic order sets did not improve the appropriateness of antibiotic doses compared with all other order types given the high rates of appropriate orders in the preimplementation period. More than $90 \%$ of dosing was appropriate in the preimplementation period at the pediatric hospital, where a paper order set reflecting antimicrobial stewardship team recommendations was required prior to transition to electronic ordering. At other hospitals, our findings were that other forms of dosing guidance were available at each hospital in the preimplementation period, which could have increased the rates of appropriateness in the preimplementation period. Though not statistically significant, electronic order sets at the pediatric hospital increased appropriateness to approximately 95\%. The increase could be related to additional decision support provided in the electronic format as compared with the paper format. These findings are in contrast to the low initial rates of appropriateness in studies of antibiotic dosing with electronic order sets in adult patients. ${ }^{15,16}$ Hall et al observed an increase from $45.5 \%$ dosing appropriateness to $67.4 \%$ when they instituted an electronic order set for vancomycin dosing, which changed the practice from standard 1-g vancomycin doses to body weight based dosing. ${ }^{16}$ Similarly, Tran et al found that the percentage of patients receiving at least $8 \mathrm{mg} / \mathrm{kg}$ of daptomycin increased from 4 to $52 \%$ following the implementation of a daptomycin dosing protocol. $^{15}$

Prior to the availability of electronic order sets, the paper order sets were mandatory for use at our pediatric tertiary care hospital. At the other system hospitals, prescribers could choose to use either a blank order sheet or the paper order set for antibiotics. It was somewhat unexpected that when specifically evaluating the nonpediatric hospitals, there was no statistically significant increase in appropriate antibiotic dosing when comparing the use of an electronic antibiotic order set to blank order sheets. The difference in appropriateness was approximately $4 \%$ between the electronic order set and the blank form, but the difference was not statistically significant. It is possible that even in the after period, prescribers found it easier to place orders without the use of the electronic order sets, but they did use the dosing provided even when ordering through nonorder set electronic methods. It is also possible that optimal dosing regimens were more frequently prescribed as time passed. Prescribers often practice at multiple system hospitals, and informal expertise sharing may occur through prescriber communication. In the pediatric hospital, where clinical pharmacists round with many medical teams, verbal dosing recommendations may have been provided to prescribers.

The two most commonly ordered antibiotics in our study, gentamicin and piperacillin-tazobactam, were dosed appropriately in more than $80 \%$ of cases even when the electronic order sets were not used, making a significant improvement difficult to demonstrate. Despite this, piperacillin-tazobactam appropriateness was approximately $7 \%$ higher with the use of the electronic order sets compared with blank order forms, though this did not reach statistical significance.

Cefepime orders were more appropriate when ordered through the electronic antibiotic order set, primarily due to the use of dosing every 12 hour regimens when dosing every 8 hours is preferable. Because there is less experience with cefepime in pediatrics than the other antibiotics, prescribers may have less familiarity with its dosing. Additionally, every 12-hour regimens of cefepime remain common in tertiary dosing references despite questionable pharmacokinetic and pharmacodynamic optimization. ${ }^{26}$ Tobramycin orders were significantly more appropriate when ordered through the electronic antibiotic order set versus a blank order sheet, which could indicate less prescriber familiarity despite similarity in dosing to gentamicin.

Implementation of CPOE can have unintended consequences. It has been shown to facilitate 22 types of medication error risks, which were related to system information errors and human-machine interface flaws. ${ }^{19}$ Han et al reported a significant increase in mortality after CPOE implementation, although this finding was not duplicated at similar centers that used the same technology and EMR. ${ }^{20,27,28}$ In fact, the subsequent studies illustrated the use of order sets and saw a decline in mortality. ${ }^{27,28}$ This study did not encounter any 
instances of similar unintended consequences. It focused on the dosing regimen only, as compared with deeper investigations into the timing of antibiotic receipt or documentation of indications, as other studies have done, and which might result in more profound effects. ${ }^{11,12,18}$

Though some inappropriate orders were observed following order set implementation in this study, it is reassuring that appropriate antibiotic dosing did not decrease. Even with the use of electronic order sets, approximately $10 \%$ of antibiotic orders were still inappropriate. This could be because of the complicated nature of patient care, which still requires additional thought beyond the support provided by any standard dosing guide or due to the need for improvement on the electronic order sets. Baysari et al performed structured interviews with prescribers after the implementation of electronic order sets, which provided useful information. ${ }^{13}$ This endeavor would have potentially shown benefit in our study, but interviews were not conducted. It was demonstrated in the same study that when the number of prewritten order sentences increased (i.e., more choices), the appropriateness of antibiotics decreased. ${ }^{13}$ A closer look at the ease of use and number of choices on our order sets is warranted. It is also possible that retrospective evaluation of appropriateness was not accurate, given the difficulty of finding all necessary information in chart review.

The health system evaluated in our study is composed of many system hospitals that differ in patient population seen and type of health care provider available. Each system hospital has differences in practice and variations in culture, which is, in part, reflected by the variability in the type of order format used at each hospital. It is difficult to assess how and when stewardship initiatives reach prescribers at system hospitals. There was no specific outreach by the stewardship program at the time of the order set availability.

Evaluation of all antibiotic orders was not possible in this study due to limited resources, but it could provide additional insights. Evaluation of appropriate antibiotic dosing for less commonly used agents, particularly newer drugs or those with unclear dosing recommendations, may demonstrate a larger impact with the use of electronic order sets. Perhaps interventions such as ours would be even more successful and demonstrate statistically significant differences if the intervention targeted at antibiotics or drugs for which evidence of inadequate dosing already exists. While the sample size of antibiotic was small, it is thought to be representative of the specific antibiotics evaluated.

Despite this, we did demonstrate adoption of the available electronic order sets at many of the hospitals, with nearly $50 \%$ of the evaluated antibiotic orders being placed through electronic order set. Specific implementation plans and education at each hospital may have further improved uptake and dosing appropriateness. Though doses ordered from the electronic order sets are only as good as the information contained in them, they were updated regularly when new primary literature or tertiary recommendations were made available.

Unfortunately, due to the high initial rate of dosing appropriateness, our study was underpowered to detect a true difference in appropriateness. Additionally, the inherent limitations of preset criteria for appropriateness, chart documentation, and the variable availability of certain information could have affected our results. The retrospective nature of our study made it difficult to understand all prescriber considerations at the time of antibiotic regimen formulation. As previously mentioned, prescribers may consult copies of the antibiotic order sets and refer to them for dosing guidance but ultimately enter their orders in a different manner such that chart documentation does not reflect the dosing guidance used at the time of prescribing. Renal function was only evaluated in instances when dosing was found to have been potentially adjusted. It is possible that some orders that should have been renally adjusted but were not were inaccurately classified as appropriate, but this situation would have occurred rarely and affected both groups equally. Finally, the system hospitals evaluated were heterogeneous; therefore, our findings are limited to the general, and more specific differences between and among hospitals may have been noted with a closer look at each hospital.

\section{Conclusion}

In this cohort, the overall percentage of orders with appropriate dose and frequency was similar before and after implementation of an electronic order set. However, the appropriateness increased slightly when comparing the use of the electronic order sets to blank order forms. Similarities were observed between the pediatric tertiary care and systems hospitals as well. Limited benefit was observed with specific antibiotics such as tobramycin and cefepime.

This study highlights a need for further investigation regarding the impact of electronic antibiotic order sets. For now, given the amount of time and effort required in the creation of the order sets, it may be prudent for stewardship or informatics departments with limited resources to direct their efforts to antibiotics with high opportunities for improvement. Simple sharing of information resources did not have a big impact on practices at affiliate system hospitals. Specific initial gap analysis to be used in the design of EHR initiatives should be evaluated. Additionally, investigation into the impact of appropriate antibiotic dosing on the incidence of adverse reactions or reduction in health care costs may contribute to our knowledge of CPOE and clinical decision support benefits.

\section{Clinical Relevance Statement}

As technology improves the rapidity and ease of sharing resources between and within health systems, clinicians and information specialists should have a plan for implementing shared resources. Required electronic order set implementation was successful at a tertiary pediatric care hospital. Optional electronic order set uptake for pediatrics in nonpediatric-focused system hospitals was successful, with nearly $50 \%$ of reviewed antibiotic orders being placed through the new electronic order sets. When it comes to dosing guidance specifically, we learned from this study that 
the optimal target for dosing improvement efforts should likely not be the antibiotics that are already being dosed appropriately. At this time, medical practitioners' critical thinking processes remain necessary in providing optimal antibiotic dosing for pediatric patients.

\section{Multiple Choice Questions}

1. Which of the following scenarios may result in a greater impact of electronic order sets on dosing appropriateness? a. A high percentage of appropriate dosing at baseline. b. A low percentage of appropriate dosing at baseline.

c. Making electronic order sets optional.

d. Requiring electronic order sets.

Correct Answer: The correct answer is option b. In this study, we found no significant difference in the appropriateness of dosing before and after electronic order sets were available. They were optional at some hospitals and required at one hospital, but this was not compared with the likelihood of appropriate dosing. Due to already high rates of dosing appropriateness though, any impacts were small and not statistically significant. Medications that are known to be dosed inappropriately may be the best target for electronic dosing guidance.

2. Approximately what percentage of orders was placed through the electronic order sets at the nonpediatric hospitals in the period after electronic order sets were available?
a. $24 \%$.
b. $50 \%$.
c. $73 \%$.
d. $98 \%$.

Correct Answer: The correct answer is option b. About half of all orders reviewed in the "after" availability period were placed through the electronic order sets, demonstrating the use of these tools. The use was higher in the pediatric hospital (nearly 100\%), but the use of the order sets was required.

3. Electronic order sets were found to improve appropriateness dosing only in comparison to which specific type of order?
a. Blank order form.
b. Nonorder set electronic orders.
c. Verbal orders.
d. Written antibiotic order sets.

Correct Answer: The correct answer is option a. When compared with orders placed on blank order forms, orders placed on electronic antibiotic order sets were more appropriate. This was not true when comparing electronic order sets to any other type of orders. Verbal orders were specifically excluded from the study.

\section{Protection of Human and Animal Subjects}

This study was performed in compliance with the World Medical Association Declaration of Helsinki on Ethical Principles for Medical Research Involving Human Subjects and was approved by Indiana University Institutional Review Board.

\section{Conflict of Interest}

Kristen Nichols is a pediatric contributor to LexiComp, the resource used to assess pediatric antibiotic dosing in this study. Otherwise, the authors report no competing interests related to this study.

\section{References}

1 Fortescue EB, Kaushal R, Landrigan CP, et al. Prioritizing strategies for preventing medication errors and adverse drug events in pediatric inpatients. Pediatrics 2003;111(4 Pt 1):722-729

2 Doherty C, Mc Donnell C. Tenfold medication errors: 5 years' experience at a university-affiliated pediatric hospital. Pediatrics 2012;129(05):916-924

3 Zed PJ, Black KJ, Fitzpatrick EA, et al. Medication-related emergency department visits in pediatrics: a prospective observational study. Pediatrics 2015;135(03):435-443

4 Stultz JS, Nahata MC. Computerized clinical decision support for medication prescribing and utilization in pediatrics. J Am Med Inform Assoc 2012;19(06):942-953

5 Rooholamini SN, Clifton H, Haaland W, et al. Outcomes of a clinical pathway to standardize use of maintenance intravenous fluids. Hosp Pediatr 2017;7(12):703-709

6 Cassel-Choudhury G, Beal J, Longani N, Leone B, Rivera R, Katyal C. Protocol-driven management of convulsive status epilepticus at a tertiary children's hospital: a quality improvement initiative. Pediatr Crit Care Med 2019;20(01):47-53

7 Hendrickson MA, Wey AR, Gaillard PR, Kharbanda AB. Implementation of an electronic clinical decision support tool for pediatric appendicitis within a hospital network. Pediatr Emerg Care 2018; 34(01):10-16

8 Luk LJ, Mosen D, MacArthur CJ, Grosz AH. Implementation of a pediatric posttonsillectomy pain protocol in a large group practice. Otolaryngol Head Neck Surg 2016;154(04):720-724

9 Hoehn EF, Overmann KM, Fananapazir N, et al. Improving emergency department care for pediatric victims of sexual abuse. Pediatrics 2018;142(06):e20181811

10 Kandel CE, Gill S, McCready J, Matelski J, Powis JE. Reducing coadministration of proton pump inhibitors and antibiotics using a computerized order entry alert and prospective audit and feedback. BMC Infect Dis 2016;16:355

11 Ozkaynak M, Wu DTY, Hannah K, Dayan PS, Mistry RD. Examining workflow in a pediatric emergency department to develop a clinical decision support for an antimicrobial stewardship program. Appl Clin Inform 2018;9(02):248-260

12 Fargo EL, D’Amico F, Pickering A, Fowler K, Campbell R, Baumgartner M. Impact of electronic physician order-set on antibiotic ordering time in septic patients in the emergency department. Appl Clin Inform 2018;9(04):869-874

13 Baysari MT, Del Gigante J, Moran M, et al. Redesign of computerized decision support to improve antimicrobial prescribing. A controlled before-and-after study. Appl Clin Inform 2017;8(03):949-963

14 Stultz JS, Nahata MC. Complexities of clinical decision support illustrated by pediatric dosing alerts. Ann Pharmacother 2015;49 (11):1261-1264

15 Tran TT, Palmer HR, Weston J, et al. Evaluation of a daptomycin dose-optimization protocol. Am J Health Syst Pharm 2012;69(11): 979-984

16 Hall AB, Montero J, Cobian J, Regan T. The effects of an electronic order set on vancomycin dosing in the ED. Am J Emerg Med 2015; 33(01):92-94

17 Simpao AF, Ahumada LM, Larru Martinez B, et al. Design and implementation of a visual analytics electronic antibiogram with 
an electronic health record system at a tertiary pediatric hospital. Appl Clin Inform 2018;9(01):37-45

18 Gunn LR, Tunney R, Kelly K. Nonmodal clinical decision support and antimicrobial restriction effects on rates of fluoroquinolone use in uncomplicated infections. Appl Clin Inform 2018;9(01):149-155

19 Koppel R, Metlay JP, Cohen A, et al. Role of computerized physician order entry systems in facilitating medication errors. JAMA 2005; 293(10):1197-1203

20 Han YY, Carcillo JA, Venkataraman ST, et al. Unexpected increased mortality after implementation of a commercially sold computerized physician order entry system. Pediatrics 2005;116(06): 1506-1512

21 Brown DM, Knoderer C. Evaluation of a Preprinted Pediatric Antimicrobial Orderset at a Free Standing Children's Hospital. ASHP Summer Meeting 2012;(JUN):51. International Pharmaceutical Abstracts, EBSCOhost. Accessed June 25, 2015

22 Webber EC, Warhurst HM, Smith SS, Cox EG, Crumby AS, Nichols KR. Conversion of a single-facility pediatric antimicrobial stewardship program to multi-facility application with computerized provider order entry and clinical decision support. Appl Clin Inform 2013;4(04):556-568
23 Lexi-Comp OnlineTM. Lexi-Drugs Online. Hudson, OH: Lexi-Comp Inc. Available at: https://online.lexi.com/lco/action/home. Accessed February 15, 2016

24 Aronoff GR, Bennett WM, Berns JS, et al. Drug Prescribing in Renal Failure. 5th ed. Philadelphia, PA: American College of Physicians; 2007

25 Conroy S, Sweis D, Planner C, et al. Interventions to reduce dosing errors in children: a systematic review of the literature. Drug Saf 2007;30(12):1111-1125

26 Shoji K, Bradley JS, Reed MD, van den Anker JN, Domonoske C, Capparelli EV. Population pharmacokinetic assessment and pharmacodynamic implications of pediatric cefepime dosing for susceptible-dose-dependent organisms. Antimicrob Agents Chemother 2016;60(04):2150-2156

27 Longhurst CA, Parast L, Sandborg CI, et al. Decrease in hospitalwide mortality rate after implementation of a commercially sold computerized physician order entry system. Pediatrics 2010;126 (01):14-21

28 Del Beccaro MA, Jeffries HE, Eisenberg MA, Harry ED. Computerized provider order entry implementation: no association with increased mortality rates in an intensive care unit. Pediatrics 2006;118(01):290-295 